DFPD 00/EP/49

ICARUS/TM-2000/04

October 31, 2018

\title{
The flavor of neutrinos in muon decays at a neutrino factory and the LSND puzzle
}

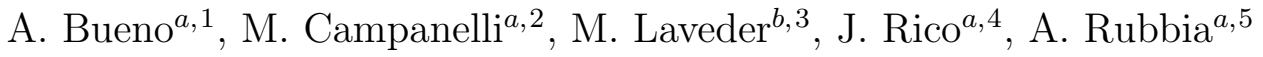 \\ ${ }^{a}$ Institut für Teilchenphysik, ETHZ, CH-8093 Zürich, Switzerland \\ ${ }^{b}$ Dip. di Fisica "G. Galilei”, Univ. di Padova and INFN Sez. di Padova, I-35131 \\ Padova, Italy
}

\begin{abstract}
The accurate prediction of the neutrino beam produced in muon decays and the absence of opposite helicity contamination for a particular neutrino flavor make a future neutrino factory the ideal place to look for the lepton flavor violating (LFV) decays of the kind $\mu^{+} \rightarrow e^{+} \bar{\nu}_{e} \nu_{\mu}$ and lepton number violating (LNV) processes like $\mu^{-} \rightarrow e^{-} \nu_{e} \nu_{\mu}$. Excellent sensitivities can be achieved using a detector capable of muon and/or electron identification with charge discrimination. This would allow to set experimental limits that improve current ones by more than two orders of magnitude and test the hypothesis that the LSND excess is due to such anomalous decays, rather than neutrino flavor oscillations in vacuum.
\end{abstract}

\footnotetext{
${ }^{1}$ Antonio.Bueno@cern.ch

${ }^{2}$ Mario.Campanelli@cern.ch

${ }^{3}$ Marco.Laveder@pd.infn.it

${ }^{4}$ Javier.Rico@cern.ch

${ }^{5}$ Andre.Rubbia@cern.ch
} 


\section{Introduction}

The Standard electroweak Model (SM) is built on an absolute conservation of three separate lepton flavors (LF): the electron flavor $L_{e}$, the muon flavor $L_{\mu}$, and the tau flavor $L_{\tau}$. The total lepton number is defined as $L_{t o t}=L_{e}+L_{\mu}+L_{\tau}$.

In a model independent way, the main decay mode of the positive muon円 into a positron and two neutrinos can be written as[1, 2]:

$$
\mu^{+} \rightarrow e^{+}+n+n^{\prime}
$$

where $n$ and $n^{\prime}$ denote neutrinos, which can be either neutrinos or antineutrinos and of any flavor $e, \mu$ or $\tau$. In the SM, individual LF conservation implies that $n \equiv \nu_{e}$ and $n^{\prime} \equiv \bar{\nu}_{\mu}$.

The evidence from neutrino oscillation searches that the neutrinos are in fact massive and mixed, implies that the LF conservation is not exact. However, LF conservation is constrained by stringent experimental limits obtained in processes involving charged leptons. For example, the present experimental 90\%C.L. upper limits on the most interesting of these decays are:

$$
\begin{aligned}
& B R(\mu \rightarrow e \gamma)<1.2 \times 10^{-11} \quad: \quad[3] \\
& B R\left(\mu^{+} \rightarrow e^{+} e^{+} e^{-}\right)<1.0 \times 10^{-12} \text { : 任 } \\
& R\left(\mu^{-} T i \rightarrow e^{-} T i\right)<6.1 \times 10^{-13}: \text { : 5] } \\
& B R(\tau \rightarrow \mu \gamma)<1.1 \times 10^{-6}: \text { : } 6
\end{aligned}
$$

These stringent limits are not inconsistent with the neutrino oscillation results since given reasonable upper bounds on the neutrino masses, the effect induced within the SM would be too small to be seen[0]. However, within extensions of the SM, neutrino oscillations raised the possible prospect that there might exist observable processes that violate the charged-lepton number [8]. Projects are currently underway to improve several of these upper limits significantly.

In contrast to this, direct experimental limits on LF conservation in decays involving neutrinos are much less stringent. For example, the limit

$$
B R\left(\mu^{-} \rightarrow e^{-} \nu_{e} \bar{\nu}_{\mu}\right)<1.2 \times 10^{-2}:[9]
$$

is orders of magnitude worse than limits involving charged leptons. In extensions of the SM, the existence of LF violation could open new modes of decay where $n$ and $n^{\prime}$ in Eq. (11) are indeed any given neutrinos or antineutrinos.

One would clearly like to experimentally test these limits by a sensitive study of the neutrinos produced in such decays. Such studies could directly be relevant to a further understanding of the neutrino sector, as described in the following sections and would represent complementary investigations to those for neutrino flavor oscillations.

If the excess of events found in the Los Alamos LSND experiment 110, 11] is interpreted as due to $\mu^{+} \rightarrow e^{+}+\bar{\nu}_{e}+n$ (with a branching ratio equal to the

\footnotetext{
${ }^{1}$ Similar arguments hold for negative muons.
} 
measured probability of $\bar{\nu}_{e}$ appearance in case of flavor neutrino oscillations), this anomalous decay could be easily tested at a future neutrino factory with a detector capable of charge discrimination, looking at the more convenient decay $\mu^{-} \rightarrow e^{-} \nu_{e} \nu_{\mu}$ as described in section 7 .

Good sensitivities could also be reached at a neutrino factory for the search

$\mu^{+} \rightarrow e^{+} \bar{\nu}_{\ell} \nu_{\mu}$ described in section 6 in which one look for interactions of $\nu_{\mu}$ giving $\mu^{-}$in a pure beam that produces $\mu^{+}$.

\section{The neutrino oscillation sector}

Hints that neutrinos are massive particles come from the observation of three anomalous effects - the LSND excess 10, 12, 11], the atmospheric anomaly 13, 14, 15, 16, 17] and the solar neutrino deficit [18, 19, 20, 21, 22]. In particular, the atmospheric results are the most convincing ones. All three effects can be naturally explained in terms of neutrino flavor oscillations, which will occur when neutrinos propagate through space, if their masses are non-degenerate and the weak and mass eigenstates are mixed 23].

However, in order to explain all three experimentally observed effects in terms of neutrino flavor oscillations, one is forced to invoke additional sterile neutrino states [24] to accommodate the very different frequencies of oscillations - given by the mass differences squared $\Delta m^{2}$ 's - indicated by the three different effects. The existence of such neutrinos is a currently unresolved problem and clearly demonstrates that the neutrino sector is not fully understood. Several attempts were made to explain all data in terms of only three massive neutrinos 25. But they are all excluded by the latest data.

From a phenomenological point of view, we recall that the neutrino flavor oscillation hypothesis predicts a well defined dependence of the phenomenon as a function of the neutrino energy, characterized by the so-called $L / E$ behavior, where $L$ is the distance between the source and detector and $E$ the neutrino energy. So far, no experiment has conclusively demonstrated such a $L / E$ dependence of the anomalous effect, with maybe the exception of the SuperKamiokande data which favors 26] a dependence $\propto L E^{n}$ where $n \approx-1$.

In such an unclear situation, is it possible to envisage "non-flavor-oscillation" mechanisms to explain part of the neutrino data?

Aside from theoretical arguments against sterile neutrinos, we argue that, from a phenomenological point of view, the LSND effect is particular: it has a small probability, measured to be $(2.5 \pm 0.6 \pm 0.4) \times 10^{-3}$ [11], in contrast to the solar and atmospheric neutrino anomalies, which are large. Hence, LSND is a natural candidate for an interpretation involving a different physics than in atmospheric and solar neutrino flavor oscillations.

\section{Relevance to the LSND puzzle}

We recall that the LSND effect was first reported as an excess of $\bar{\nu}_{e}$ 's in the $\bar{\nu}_{\mu}$ 's flux from the $\mu^{+}$Decay-At-Rest (DAR) process 10. The neutrino beam 
is obtained with $800 \mathrm{MeV}$ kinetic energy protons hitting a series of targets, producing secondary pions. Most of the $\pi^{+}$come to rest and decay through the sequence $\pi^{+} \rightarrow \mu^{+} \nu_{\mu}$, followed by $\mu^{+} \rightarrow e^{+} \nu_{e} \bar{\nu}_{\mu}$, supplying the experiment with the $\bar{\nu}_{\mu}$ 's with a maximum energy of $52.8 \mathrm{MeV}$. The intrinsic contamination of $\bar{\nu}_{e}$ 's coming from the symmetrical decay chain starting with $\pi^{-}$is estimated to be small since most negatively charged mesons are captured before they decay.

The excess of $\bar{\nu}_{e}$ 's, explained in terms of neutrino flavor transitions of the type $\bar{\nu}_{\mu} \rightarrow \bar{\nu}_{e}$, occurs via the reactions:

$$
\mu^{+} \rightarrow e^{+} \nu_{e} \bar{\nu}_{\mu} ; \quad \bar{\nu}_{\mu} \stackrel{\text { vacuum }}{\longrightarrow} \bar{\nu}_{e} ; \quad \bar{\nu}_{e} p \rightarrow e^{+} n
$$

Additional evidence in favor of neutrino oscillation was reported in the DecayIn-Flight (DIF) sample, though with a low statistical significance 12]. In a recent re-analysis of the complete data sample collected[11], the significance of the DIF data seems to be even lower. Hence, we concentrate on the hint from stopped muons, and ignore the DIF result.

The latest KARMEN2 results 27 come very close to contradicting the LSND claim, however the experimental sensitivity is marginal to conclusively exclude or confirm completely the LSND excess. A new experiment, MiniBOONE[28], will confront the flavor oscillation hypothesis with a very high statistical accuracy.

In case of a negative result, one will only be able to conclude that the LSND excess was not due to neutrino flavor oscillations.

The implications of exotic muon decays on the LSND excess has been studied 29] showing that two explicit models predicted interactions of about one order of magnitude smaller than what would be relevant for LSND. In a model independent proof in which any contributions from neutrino mixing is neglected, the authors of Ref. [30] prove that new lepton flavor violating interactions, under the constraint of LF data involving charged leptons, fail short to explain the LSND effect by a rate factor of almost three. However in Ref. [31], it is reported that exotic decays that produce two antineutrinos

$$
\mu^{+} \rightarrow e^{+}+\bar{\nu}_{e}+\bar{\nu}_{\ell} \quad(\ell=e, \mu, \tau)
$$

cannot be ruled out as the cause of the LSND excess in a model independent way.

Regardless of any theoretical prejudice, the excess of electrons found by the LSND experiment stands today as a still unresolved puzzle of neutrino physics.

\section{Searches at a neutrino factory}

A neutrino factory [32, 33] is understood as a machine where low energy muons of a given charge are accelerated in a storage ring. The two neutrinos $n, n^{\prime}$ produced in the decay Eq. (四) will be boosted in the forward direction of the muon flight path. Hence, the muon storage ring is composed of long straight-lines in order to produce directional neutrino beams.

In such machines, muons are produced in decays of secondary pions produced by few $\mathrm{GeV}$ protons incident on a target. In current designs, muons are captured 
with high efficiency and very high integrated protons-on-target intensities are envisaged in order to produce very intense neutrino sources.

The neutrino physics potentialities of such machines has been largely discussed in the literature 34, 35]. In particular, we mention our study in the context of a short-baseline experiment to search for neutrino flavor oscillation in a background free environment in the $\Delta m^{2}$ region indicated by LSND 36].

A neutrino factory is also an ideal place to study neutrinos from exotic muon decays. The envisaged flux of neutrinos is sufficiently high to obtain large statistics of neutrino interaction events. More importantly, we can take advantage from the fact that the neutrino beam is produced from muons of a definite sign and therefore the decay processes can be studied with a pure initial state. This is not the case for traditional pion decay neutrino beams, in which contaminations are always present at some level.

The flavor of the interacting neutrinos can be tested via their charged current processes. In case of purely lepton flavor conserving decays $\mu^{+} \rightarrow e^{+} \nu_{e} \bar{\nu}_{\mu}$, we expect to detect only

$$
\begin{gathered}
\nu_{e}+N \rightarrow e^{-}+X \\
\bar{\nu}_{\mu}+N \rightarrow \mu^{+}+X
\end{gathered}
$$

while exotic decays can be immediately identified by various processes

$$
\begin{array}{ll}
\mu^{+} \rightarrow e^{+}+n+\nu_{\mu} \longrightarrow & \nu_{\mu}+N \rightarrow \mu^{-}+X \\
\mu^{+} \rightarrow e^{+}+\bar{\nu}_{e}+n \longrightarrow & \bar{\nu}_{e}+N \rightarrow e^{+}+X \\
\mu^{+} \rightarrow e^{+}+n+\nu_{\tau} \longrightarrow & \nu_{\tau}+N \rightarrow \tau^{-}+X \\
\mu^{+} \rightarrow e^{+}+n+\bar{\nu}_{\tau} \longrightarrow & \bar{\nu}_{\tau}+N \rightarrow \tau^{+}+X
\end{array}
$$

where $n$ stands for neutrinos or anti-neutrinos.

Charge discrimination of electrons and muons can trivially separate the two types of decays. The presence of taus is more difficult to identify but can be achieved using topological or kinematical signatures. It requires neutrino beams of high energy in order to exceed the tau lepton production threshold. We do not consider tau identification any further in this paper, and concentrate on the identification of electrons and muons in a low energy setup.

In order to predict the energy distribution of the (anti)neutrinos in the detector we assume for definiteness two types of generic decays:

$$
\begin{aligned}
& \mu^{+} \rightarrow e^{+}+\bar{\nu}_{\ell}+\nu_{\mu} \\
& \mu^{-} \rightarrow e^{-}+\nu_{e}+\nu_{\ell}
\end{aligned}
$$

We address the search for LFV, Eq. (15), using wrong sign muons, which are experimentally simpler to detect. In order to profit from the enhanced crosssection of neutrinos versus antineutrinos, it is better to select positive muons in the storage ring, since in this case, the LFV decays produce $\nu_{\mu}$ 's.

In case we consider the LNV decay of Eq. (16), for which $\Delta L=2$, we should look for wrong sign electrons. We select $\mu^{-}$in the ring since the signal searched 
for in this case has two neutrinos in the final state, therefore we profit from the enhanced neutrino cross sections. Naturally, both signs of muons could be studied in a real experiment, in order to provide possible checks for different behaviors in $\mu^{+}$or $\mu^{-}$decays.

Following the discussion in [31], LNV interactions can naturally arise via mixing of heavy bosons that transform differently under the Standard Model group but identically under the unbroken $U(1)_{E M}$. In particular, the effective four-fermion operator relevant for the reaction:

$$
\mu_{L}^{-} \rightarrow e_{R}^{-}+\nu_{e}+\nu_{\ell}
$$

which violates lepton number conservation by two units, has the form $\left(\mu_{L} \bar{\nu}_{e}\right)\left(\bar{\nu}_{\ell} \bar{e}_{R}\right)$. It couples $\nu_{e}$ to $\mu^{-}$and $e^{-}$to $\nu_{\ell}$. This operator can be induced, for example in supersymmetric models without R-parity, through the mixing of sfermions that are $S U(2)_{L}$ singlets with sfermions that are $S U(2)_{L}$ doublets.

After explicit calculation, we obtain that the square of the scattering amplitude for the decay (17) is $\left\langle|\mathcal{M}|^{2}>\propto\left(p_{\mu} \cdot p_{\nu_{e}}\right)\left(p_{e} \cdot p_{\nu_{\mu}}\right)\right.$ (where $p_{i}$ is the four-momentum of particle $i$ ). This expression is similar to the one obtained for the standard decay $\mu^{-} \rightarrow e^{-} \bar{\nu}_{e} \nu_{\mu}$; therefore the flux of $\nu_{e}$ 's coming from (17) is equal to the one of $\bar{\nu}_{e}$ 's produced in the standard $\mu^{-}$decay as shown in Figure 1 .

\section{$5 \quad$ Experimental considerations}

We address a few experimental considerations in the context of an optimization of searches for LFV and LNV decays of the muon.

\subsection{Beam setup}

Unlike for neutrino flavor oscillations in vacuum, the distance $L$ between source and detector is in this case an irrelevant physical parameter. It is hence advantageous to place the experiment close to the source in order to gain flux like $1 / L^{2}$ due to the beam divergence.

We think that a neutrino beam energy of a few $\mathrm{GeV}$ would be optimal in order to facilitate the discrimination of the muon and electron charges, and in order to reduce misidentified electrons or muons background coming mostly from neutral current interactions. We therefore consider a low-energy muon storage ring 2 with three possible muon energies $E_{\mu}=1,2$ or $5 \mathrm{GeV}$.

In Table 1, we list the expected event rates from standard muon decays per ton of target and $10^{19}$ standard muon decays. The detector is located at a distance $L=100 \mathrm{~m}$ from a $100 \mathrm{~m}$ long straight section of the storage ring. The expected event energy spectra are shown in Figure 2 .

\footnotetext{
${ }^{2}$ We also note that studies indicate that the cost of a neutrino factory is driven by the muon energy $E_{\mu}$ and rises very rapidly with $E_{\mu}$, hence a low energy muon beam is also financially favored.
} 


\begin{tabular}{|cc|c|c|c|}
\hline \hline & & $E_{\mu}=1 \mathrm{GeV}$ & $E_{\mu}=2 \mathrm{GeV}$ & $E_{\mu}=5 \mathrm{GeV}$ \\
\cline { 3 - 5 } & $\nu_{\mu} \mathrm{CC}$ & 3300 & 23200 & 233400 \\
$\mu^{-}$ & $\nu_{\mu} \mathrm{NC}$ & 410 & 4470 & 58600 \\
$10^{19}$ decays & $\bar{\nu}_{e} \mathrm{CC}$ & 630 & 6250 & 80200 \\
& $\bar{\nu}_{e} \mathrm{NC}$ & 100 & 1450 & 23100 \\
\hline \hline \multirow{4}{*}{$\mu^{+}$} & $\bar{\nu}_{\mu} \mathrm{CC}$ & 730 & 7200 & 91200 \\
& $\bar{\nu}_{\mu} \mathrm{NC}$ & 140 & 1830 & 27600 \\
& $\nu_{e} \mathrm{CC}$ & 3060 & 21500 & 211700 \\
& $\nu_{e} \mathrm{NC}$ & 310 & 3660 & 50100 \\
\hline
\end{tabular}

Table 1: Expected event rates $(\mathrm{CC}=$ charged current, $\mathrm{NC}=$ neutral currents) per ton of target per $10^{19}$ standard muon decays in a storage ring with a straight line of $100 \mathrm{~m}$ and located $100 \mathrm{~m}$ away from the neutrino detector. Only $50 \%$ of the muons are assumed to decay in the direction of the detector, the other $50 \%$ are lost.

\subsection{Detector parameters}

Experimentally, the presence of LFV decays will characterize themselves by the observation of events with "wrong sign leptons". It is therefore mandatory to ensure a very good and efficient determination of the lepton charge.

The muon charge is most easily determined with the help of bending in a magnetic field. The radius of curvature in meters in a $1 \mathrm{~T}$ field is approximately $10 p(\mathrm{GeV}) / 3$, or 3 meters for $p=1 \mathrm{GeV}$. In the case of electrons, the capability to measure the charge is limited by the radiation length of the target which determines the distance after which the electromagnetic shower develops at a level where the primary electron is not distinguishable any longer.

At low energies, we expect events to exhibit simple topologies and many of them will be quasi-elastic-like. Hence, the events will be dominated by a leading hard lepton accompanied by few soft hadrons. Such events were traditionally best studied in bubble chambers, due to the required low density, high granularity and homogeneity to capture soft escaping tracks at all angles. In addition, a low density, high granularity instrumented target is mandatory to efficiently recognize electrons and to discriminate muons from pions. Different detector configurations meeting the previously mentioned specifications can be clearly envisaged.

\section{Results for $\mu^{+} \rightarrow e^{+}+\bar{\nu}_{\ell}+\nu_{\mu}$}

We consider a 10 ton fiducial mass detector located at a distance of $100 \mathrm{~m}$ from the muon storage ring.

For a $2 \mathrm{GeV}$ muon ring energy, the expected event samples for a total of $10^{19}$ standard $\mu^{+}$decays are 72'000 $\bar{\nu}_{\mu}$ CC and 215'000 $\nu_{e}$ CC events, and 18'300 $\bar{\nu}_{\mu}$ $\mathrm{NC}$ and $36{ }^{\prime} 600 \nu_{e} \mathrm{NC}$.

To estimate the signal efficiency, we assume that the LFV decay proceeds 


\begin{tabular}{|c|c|c|c|c|}
\hline Cuts & $\bar{\nu}_{\mu} \mathrm{CC}$ & $\bar{\nu}_{\mu} \mathrm{NC}$ & $\nu_{e} \mathrm{NC}$ & LFV $\nu_{\mu} \mathrm{CC}$ \\
\hline \hline \multicolumn{5}{|c|}{$E_{\mu^{+}}=2 \mathrm{GeV}$} \\
\hline Initial & 72000 & 18300 & 36600 & 540 \\
$\mu^{-}$candidate & 9890 & 660 & 1197 & 540 \\
$E_{\mu^{-}}>1.1 \mathrm{GeV}$ & $<0.1$ & 0.6 & 2 & 130 \\
\hline \hline \multicolumn{5}{|c|}{$E_{\mu^{+}}=5 \mathrm{GeV}$} \\
\hline Initial & 912000 & 276000 & 501000 & 5290 \\
$\mu^{-}$candidate & 133500 & 10500 & 17900 & 5290 \\
$E_{\mu^{-}}>3 \mathrm{GeV}$ & 3 & 0.3 & 0.6 & 645 \\
\hline \hline \multicolumn{5}{|c|}{$E_{\mu^{+}}=1 \mathrm{GeV}$} \\
\hline Initial & 7300 & 1400 & 3100 & 76 \\
$\mu^{-}$candidate & 873 & 46 & 99 & 76 \\
$E_{\mu^{-}}>0.6 \mathrm{GeV}$ & $<0.1$ & 0.1 & 0.6 & 16 \\
\hline \hline
\end{tabular}

Table 2: Effect of cuts on background and signal. We assumed a positive muon ring energy $E_{\mu^{+}}$of 1,2 and $5 \mathrm{GeV}$ and a total of $10^{19}$ standard decays. The LFV decay has the branching probability of $2.5 \times 10^{-3}$. Backgrounds come from hadrons escaping the detector without interacting or muons from meson decays. For the charged current background, no veto on the positive muon has been included. $E_{\mu^{-}}$is the energy of the identified negative muon in the event.

through a similar diagram as the standard muon decay as given by the $V-A$ theory, however, with interchanged neutrino flavors. We can then essentially assume that at the detector location the flux of $\nu_{\mu}$ 's from LFV decays is similar to the one of $\nu_{e}$ 's in the standard muon decay. It is clear that other type of interactions could be envisaged and could lead to different energy spectra which can be experimentally tested by studying the visible energy distribution of wrong sign muon events.

If LFV decay occur with a branching $B r(L F V)$, we expect to observe the number of negative muon events

$$
\begin{aligned}
N_{\mu^{-}, C C} & =\int \Phi_{L F V}\left(\nu_{\mu}, E_{\nu}\right) \sigma^{C C}\left(\nu_{\mu}, E_{\nu}\right) d E_{\nu} \\
& \approx \operatorname{Br}(L F V) \times \int \Phi\left(\nu_{e}, E_{\nu}\right) \sigma^{C C}\left(\nu_{\mu}, E_{\nu}\right) d E_{\nu}
\end{aligned}
$$

where $\Phi_{L F V}$ is the flux of $\nu_{\mu}$ neutrinos from LFV decays, $\Phi\left(\nu_{e}\right)$ is the flux of electron neutrinos in standard $\mu^{+}$decays and $\sigma^{C C}$ the charged current crosssections.

For the branching indicated by LSND and $10^{19} \mu^{+}$decays, we obtain

$$
\begin{aligned}
N_{\mu^{-}, C C} & \approx 215^{\prime} 000 \times B r(L F V) \\
& =540
\end{aligned}
$$

for the fiducial detector mass of 10 ton. 
For muon tracks of a few $\mathrm{GeV}$ momentum bent in the magnetic field, we expect wrong charge confusion at the level of $\approx 10^{-3 \%}$. We therefore think that the background produced by $\bar{\nu}_{\mu} \mathrm{CC}$ events with mismeasured charge of the muon to be less than one event.

One crucial experimental aspect is the discrimination of muons versus pions. In addition, secondary muons from meson decays produce background, especially at low energies. In order to assess realistic efficiencies and experimental backgrounds, we illustrate results with a detector with the characteristics of the ICARUS liquid Argon imaging TPC 37]. The detector would be on a mediumsized $L A r$ vessel surrounded by a dipole magnet. The size of such detector would be in the range of $2 \times 2 \times 6 \mathrm{~m}^{3}$ for a total mass of about 30 tons. The magnetic field would be oriented perpendicular to the drift $E$-field and the incoming neutrino direction, in order to bend the charged particles in the direction of the drift field, where a resolution in the range of $200 \mu \mathrm{m}$ is expected from the LAr TPC. Given the hadronic interaction length of $L A r$ of $\lambda_{I}=84 \mathrm{~cm}$, muons which loose about $240 \mathrm{MeV} / \mathrm{m}$ are distinguished from pions which interact hadronically.

The effect of simple cuts on background and signal are illustrated in Table 2 . We assumed in this case a positive muon ring energy and a total of $10^{19}$ standard decays. The LFV decay has the branching probability of $2.5 \times 10^{-3}$, compatible with the LSND excess.

We considered all sources of backgrounds by means of fully generated neutrino events. Large event samples were produced for all neutrino species with the proper energy distribution with the help of an event generator 38. which includes all exclusive final states and a realistic treatment of the low energy region.

A muon candidate is identified as a track which stops without interacting in the Argon or leaves the detector vessel before interacting or stopping. Hence, charged and neutral current backgrounds come from hadrons escaping the detector without interacting or or from actual muons from meson decays. For the charged current background, no veto on the positive muon has been included.

In Table 2, we observe that the presence of LFV decays will produce an excess of events with $\mu^{-}$candidates. The visible energy and the candidate muon energy spectra are shown in Figure 3. As expected, the excess is most visible at the highest end of the muon energy distribution, since background comes from misidentified soft hadrons. A simple cut on the muon candidate energy can be used to largely suppress background to negligible level, while keeping a large fraction of the LFV signal events, as shown in the Table 2. The visible energy distribution of events for $E_{\mu^{+}}=2 \mathrm{GeV}$ with a cut on the muon candidate momentum of $1.1 \mathrm{GeV}$ (efficiency for LFV of 25\%) is shown in Figure 4 . The statistics is clearly sufficient to constrain any theoretical prediction of the energy distribution of the LFV neutrinos.

\section{$7 \quad$ Results for $\mu^{-} \rightarrow e^{-}+\nu_{e}+\nu_{\ell}$}

The experimental signal consists in the appearance of final state electrons, while standard events have positrons in the final state. Therefore, to experimentally 


\begin{tabular}{|c|c|c|}
\hline Cuts & $\nu_{e} \mathrm{CC}$ & $\bar{\nu}_{e} \mathrm{CC}$ \\
\hline \multicolumn{3}{|c|}{$\begin{array}{l}E_{\mu^{-}}=2 \mathrm{GeV} \\
\end{array}$} \\
\hline Initial & 540 & 62500 \\
\hline One proton & 367 & 11000 \\
\hline No pions & 323 & 100 \\
\hline$E_{e}>1 \mathrm{GeV}$ & 103 & 17 \\
\hline Candidate charge & 21 & 0.4 \\
\hline \multicolumn{3}{|c|}{$\overline{E_{\mu^{-}}}=5 \mathrm{GeV}$} \\
\hline Initial & 5290 & 802000 \\
\hline One proton & 3390 & 212160 \\
\hline No pions & 2112 & 495 \\
\hline$E_{e}>3 \mathrm{GeV}$ & 351 & 163 \\
\hline Candidate charge & 71 & 4 \\
\hline \multicolumn{3}{|c|}{$E_{\mu^{-}}=1 \mathrm{GeV}$} \\
\hline Initial & 76 & 6300 \\
\hline One proton & 53 & 529 \\
\hline No pions & 48 & 8 \\
\hline$E_{e}>0.2 \mathrm{GeV}$ & 43 & 4 \\
\hline Candidate charge & 10 & 0.1 \\
\hline
\end{tabular}

Table 3: Effect of cuts on background and signal. We assumed a negative muon ring energy $E_{\mu^{-}}$of 1,2 and $5 \mathrm{GeV}$ and a total of $10^{19}$ standard decays. The lepton number violating decay has the branching probability of $2.5 \times 10^{-3} . E_{e}$ is the energy of the identified electron in the event.

detect reaction (16), it is mandatory to envisage an experiment endowed with charge discrimination capabilities for electrons. This is a true experimental challenge given the short radiation lengths in dense targets.

In order to evaluate realistic efficiencies and backgrounds, we consider an experimental setup similar to the one discussed in the previous section, namely, an ICARUS liquid Argon imaging TPC with a magnetic field provided by an external dipole magnet.

Table 3 shows the effect of the cuts applied for a normalization of $10^{19}$ muon decays. To compute the expected number of signal events we have taken a branching probability of $2.5 \times 10^{-3}$. Given the low muon energies considered, most of the events will be quasielastic. We thus require a final state configuration containing an electron and a reconstructed proton and no additional hadronic particles. These criteria reduce the quasielastic background (where we expect a neutron rather than a proton in the final state) by almost three orders of magnitude, while keeping more than $50 \%$ of the signal. The NC background, where electron candidates come from $\pi^{0}$ conversion, is in general soft. After

\footnotetext{
${ }^{3}$ We assume a kinetic threshold of $T>50 \mathrm{MeV}$ in order to detect a proton.
} 
a cut on the electron candidate momentum, this kind of background becomes negligible.

As mentioned before, charge discrimination between electrons and positrons is a must for this kind of search. Preliminary studies show 39] that for a LAr detector immerse in a $1 \mathrm{~T}$ magnetic field, a fit to the direction of the electromagnetic shower could provide a good determination of the charge of leading electrons. When loose criteria are applied, the expected charge contamination from $\bar{\nu}_{e}$ CC amounts to $2 \%$ for an electron efficiency of $25 \%$. Tighter requirements reduce the charge confusion to the per mil level for an electron identification efficiency close to $10 \%$. Table 3 shows that applying loose criteria in the determination of the lepton charge is enough to eliminate $\bar{\nu}_{e}$ CC background.

\section{Experimental sensitivities}

Table 1 shows the expected sensitivities in case a negative result is found as a function of the muon energy circulating in the accumulation ring. Three different normalizations have been considered: $10^{18}, 10^{19}$ and $10^{20}$ muons. For comparison we also show the current best limit on the LFV decay $\mu^{+} \rightarrow e^{+} \nu_{e} \bar{\nu}_{\mu}[9]$.

For a statistics corresponding to $10^{19}$ muon decays we could improve the present sensitivity by more than two orders of magnitude. Three orders of magnitude can be reached for $10^{20}$ muons. Therefore the interpretation of the LSND excess in terms of anomalous muon decay that violates lepton flavor and/or total lepton number could be thoroughly experimentally tested.

\begin{tabular}{lccccc}
\hline \hline $\begin{array}{c}\mu \text { Decays } \\
10^{18}\end{array}$ & Decay mode & Current Limit & $E_{\mu}=1 \mathrm{GeV}$ & $E_{\mu}=2 \mathrm{GeV}$ & $E_{\mu}=5 \mathrm{GeV}$ \\
\cline { 3 - 5 } $10^{19}$ & $P\left(\mu^{+} \rightarrow e^{+} \bar{\nu}_{\ell} \nu_{\mu}\right)<$ & \multirow{2}{*}{$1.2 \times 10^{-2}$} & $5 \times 10^{-3}$ & $5 \times 10^{-4}$ & $1 \times 10^{-4}$ \\
$10^{20}$ & & & $2 \times 10^{-4}$ & $1 \times 10^{-4}$ & $3 \times 10^{-5}$ \\
\hline $10^{18}$ & \multirow{3}{*}{} & & $6 \times 10^{-3}$ & $3 \times 10^{-5}$ & $2 \times 10^{-5}$ \\
$10^{19}$ & $P\left(\mu^{-} \rightarrow e^{-} \nu_{e} \nu_{\ell}\right)<$ & - & $6 \times 10^{-4}$ & $3 \times 10^{-4}$ & $9 \times 10^{-4}$ \\
$10^{20}$ & & & $1 \times 10^{-4}$ & $1 \times 10^{-4}$ & $2 \times 10^{-4}$ \\
\hline \hline
\end{tabular}

Table 4: Achievable limits in case of negative result at the $90 \%$ C.L. for $\mu^{+} \rightarrow e^{+} \bar{\nu}_{\ell} \nu_{\mu}$ and $\mu^{-} \rightarrow e^{-} \nu_{e} \nu_{\ell}$ decays with a 10 ton detector for three different number of muon decays.

\section{Conclusion}

A negative result from the MiniBOONE experiment would indicate that the neutrino flavor oscillation is not the correct hypothesis to explain the excess seen in LSND. It would however not contradict other possible non-flavor-oscillation interpretations of the effect. 
In particular, LFV and LVN decays could play a role in the interpretation of the LSND excess. A better understanding of these processes would then be particularly relevant, if not mandatory.

A neutrino factory is an ideal machine to probe such anomalous decays of the muon. The pure initial state beam allows to look for these decays without intrinsic beam contamination. 


\section{References}

[1] P. Herczeg, Z. Phys. C56, S129 (1992).

[2] P. Herczeg, Los Alamos Sci. (1997) No. 25 128-135.

[3] M.L. Brooks et al., MEGA collaboration, hep-ex/9905013.

[4] U. Bellgardt et al., Nucl. Phys. B229 (1988) 1.

[5] P. Wintz, Proceedings of the First International Symposium on Lepton and Baryon Number Violation, eds. H.V. Klapdor-Kleingrothaus and I.V. Krivosheina (Institute of Physics, Bristol, 1998), p.534.

[6] S. Ahmed et al., CLEO Collaboration, hep-ex/9910060.

[7] P. Herczeg, in Proceedings of the Zuoz Summer School on Hidden Symmetries and Higgs Phenomena, Zuoz, Switzerland, 16-22 Aug 1998.

[8] J. Ellis, M. E. Gomez, G. K. Leontaris, S. Lola and D. V. Nanopoulos, Eur. Phys. J. C14, 319 (2000) hep-ph/9911459].

[9] S. J. Freedman et al., Phys. Rev. D47 (1993) 811.

[10] C. Athanassopoulos et al. [LSND Collaboration], Phys. Rev. Lett. 77, 3082 (1996) nucl-ex/9605003; Phys. Rev. C54, 2685 (1996) nucl-ex/9605001.

[11] G. Mills [for the LSND Collaboration], Talk presented at Neutrino 2000, Sudbury, Canada, 16-21 June 2000 (http://nu2000.sno.laurentian.ca).

[12] C. Athanassopoulos et al. [LSND Collaboration], Phys. Rev. Lett. 81, 1774 (1998) nucl-ex/9709006; Phys. Rev. C58, 2489 (1998) nucl-ex/9706006.

[13] Y. Fukuda et al. (Kamiokande Collaboration), Phys. Lett. B 335, 237 (1994).

[14] R. Becker-Szendy et al. (IMB Collaboration), Nucl. Phys. B (Proc. Suppl.) 38, 331 (1995).

[15] S. Fukuda et al. (Super-Kamiokande Collaboration), hep-ex/0009001; T. Kajita (Super-Kamiokande Collaboration), Talk presented at NOW2000, Otranto, Italy, September 2000 (http://www.ba.infn.it/ now2000).

[16] W.W.M. Allison et al. (Soudan 2 Collaboration), Phys. Lett. B 449, 137 (1999).

[17] F. Ronga (MACRO Collaboration), Talk presented at NOW2000, Otranto, Italy, September 2000 (http://www.ba.infn.it/〜now2000).

[18] B. T. Cleveland et al., Astrophys. J. 496, 505 (1998).

[19] Kamiokande, Y. Fukuda et al., Phys. Rev. Lett. 77, 1683 (1996).

[20] GAlleX, W. Hampel et al., Phys. Lett. B447, 127 (1999).

[21] SAGE, J. N. Abdurashitov et al., Phys. Rev. C60, 055801 (1999), astro$\mathrm{ph} / 9907113$. 
[22] Y. Suzuki (Super-Kamiokande Coll.), Talk presented at Neutrino 2000, Sudbury, Canada, 16-21 June 2000 (http://nu2000.sno.laurentian.ca); Talk presented at NOW2000, Otranto, Italy, September 2000 http://www.ba.infn.it/ now2000).

[23] B. Pontecorvo, J. Expt. Theor. Phys. 33, 549 (1957) [Sov. Phys. JETP 6, 429 (1958)]; B. Pontecorvo, J. Expt. Theor. Phys. 34, 247 (1958) [Sov. Phys. JETP 7, 172 (1958)]; Z. Maki, M. Nakagawa and S. Sakata, Prog. Theor. Phys. 28 (1962) 870; B. Pontecorvo, J. Expt. Theor. Phys 53 (1967) 1717; V. Gribov and B. Pontecorvo, Phys. Lett. B 28, 493 (1969).

[24] see e.g. C. Giunti, Nucl. Instrum. Meth. A451, 51 (2000) hep-ph/9910336.

[25] C. Y. Cardall and G. M. Fuller, Phys. Rev. D53, 4421 (1996) astroph/9602104; A. Acker and S. Pakvasa, Phys. Lett. B397, 209 (1997) [hepph/9611423]; R. P. Thun and S. McKee, Phys. Lett. B439, 123 (1998) hep-ph/9806534.

[26] H. Sobel, [for the Super-Kamiokande Collaboration], Talk presented at Neutrino 2000, Sudbury, Canada, 16-21 June 2000 (http://nu2000.sno.laurentian.ca).

[27] K. Eitel [KARMEN Collaboration], hep-ex/0008002.

[28] see e.g. A. Bazarko [MiniBooNE Collaboration], hep-ex/0009056.

[29] P. Herczeg, in Proceedings of the Workshop on Physics Beyond the Standard Model: Beyond the Desert: Accelerator and Nonaccelerator Approaches, Tegernsee, Germany, 8-14 Jun 1997.

[30] S. Bergmann and Y. Grossman, Phys. Rev. D59, 093005 (1999) hep$\mathrm{ph} / 9809524$.

[31] S. Bergmann, H. V. Klapdor-Kleingrothaus and H. Pas, Phys. Rev. D62, 113002 (2000) hep-ph/0004048].

[32] S. Geer, Phys. Rev. D 57 (1998) 6989.

[33] Information on the neutrino factory studies and mu collider collaboration at BNL can be found at http://www.cap.bnl.gov/mumud. Information on the neutrino factory studies at FNAL can be found at http://www.fnal.gov/projects/muon_collider/. Information on the neutrino factory studies at CERN can be found at http://muonstoragerings.cern.ch/Welcome.html].

[34] C. Albright et al., hep-ex/0008064.

[35] A. de Rújula, M. B. Gavela and P. Hernández, Nucl. Phys. B 547 (1999) 21.; V. Barger, S. Geer, R. Raja and K. Whisnant, Phys. Rev. D62, 013004 (2000) hep-ph/9911524; Phys. Rev. D62, 073002 (2000) [hep-ph/0003184; A. Bueno, M. Campanelli and A. Rubbia, Nucl. Phys. B573 (2000) 27; V. Barger, S. Geer and K. Whisnant, Phys. Rev. D 61 (2000) 053004.; M. Freund, M. Lindner, S. T. Petcov and A. Romanimo, Nucl. Phys. B578, 27 (2000) hep-ph/9912457; A. Cervera et al., Nucl. Phys. B579, 
17 (2000) hep-ph/0002108; A. Bueno, M. Campanelli and A. Rubbia, hepph/0005007, accepted for publication in Nucl. Phys. B.

[36] A. Bueno, M. Campanelli and A. Rubbia, hep-ph/9809252.

[37] see C. Vignoli et al. [ICARUS Collaboration], Nucl. Phys. Proc. Suppl. 85 (2000) 119 and references therein.

[38] A. Ferrari and A. Rubbia, "NUX: a neutrino event generator", ICARUS internal note in preparation.

[39] M. Campanelli and A. Rubbia, ICARUS internal note in preparation. 


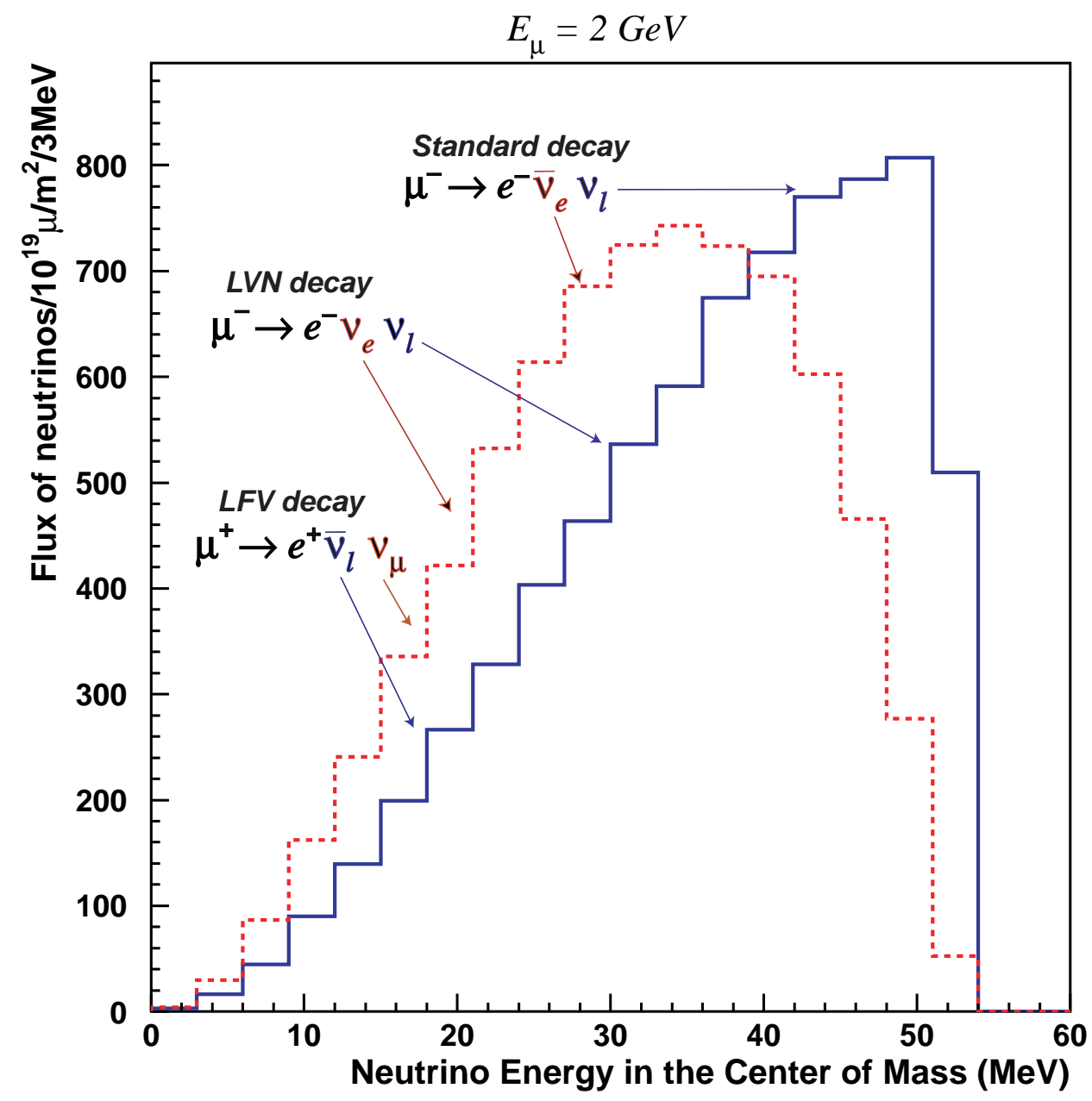

Figure 1: Expected neutrino fluxes for the standard, lepton flavor and lepton number violating $\mu^{-}$decays as a function of the neutrino energy in the center of mass reference frame. 

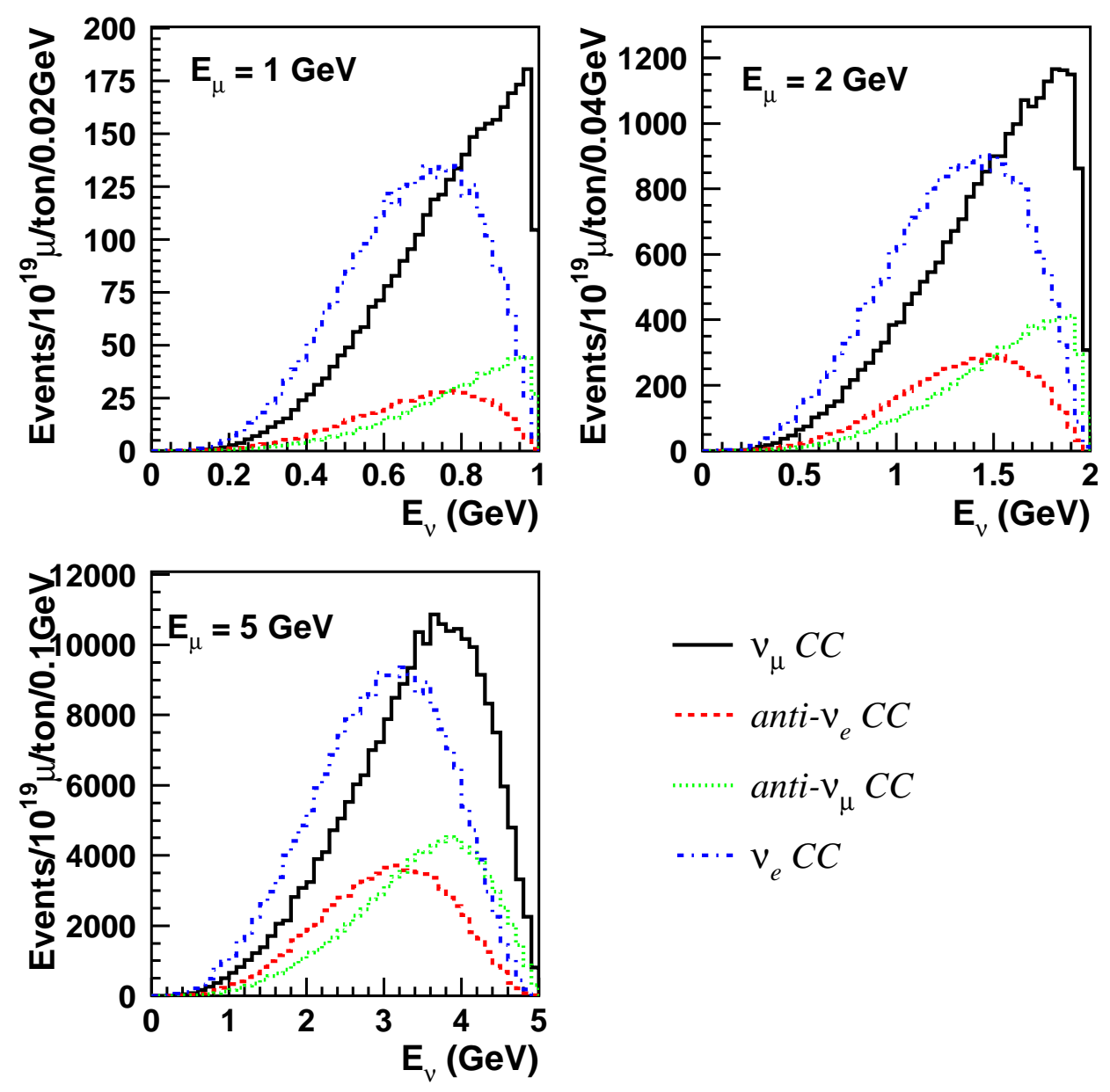

$$
\begin{aligned}
& -v_{\mu} C C \\
& \text { anti- } v_{e} C C \\
& \text { anti- } v_{\mu} C C \\
& \cdots \mathrm{v}_{e} C C
\end{aligned}
$$

Figure 2: Neutrino events energy spectrum for muon ring energy of 1,2 and $5 \mathrm{GeV}$. The $\nu_{\mu}$ (line) and $\bar{\nu}_{e}$ (dashed) events come from standard $\mu^{-}$decays, while $\bar{\nu}_{\mu}$ (dotted) and $\nu_{e}$ (dash-dotted) events come from standard $\mu^{+}$decays. 

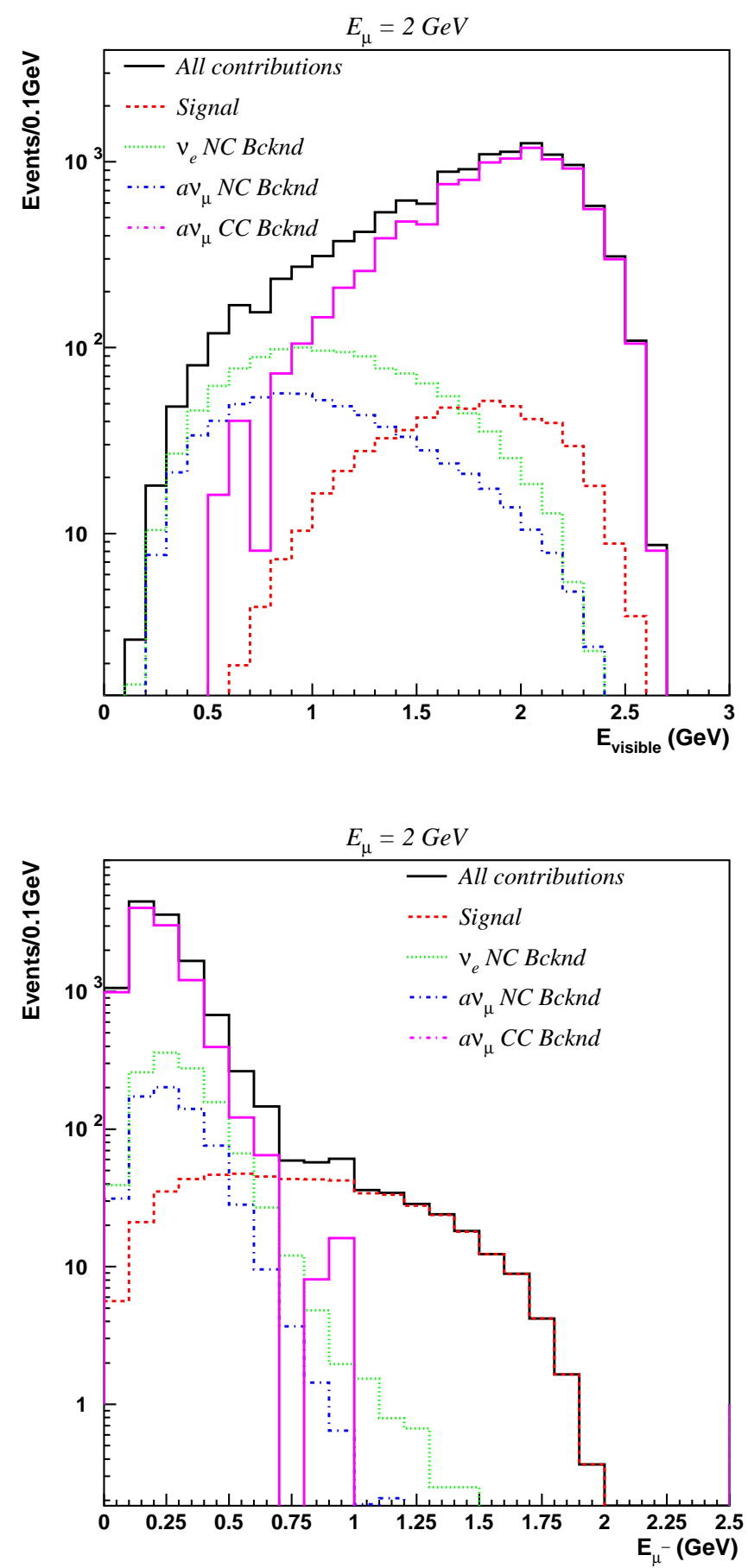

Figure 3: Visible energy (upper) and candidate muon (lower) distribution for LFV decays (see text) normalized to LSND excess and $10^{19}$ positive muon decays for 10 ton detector. The background from neutral current using the characteristics of an ICARUS $L A r$ TPC is also shown. 


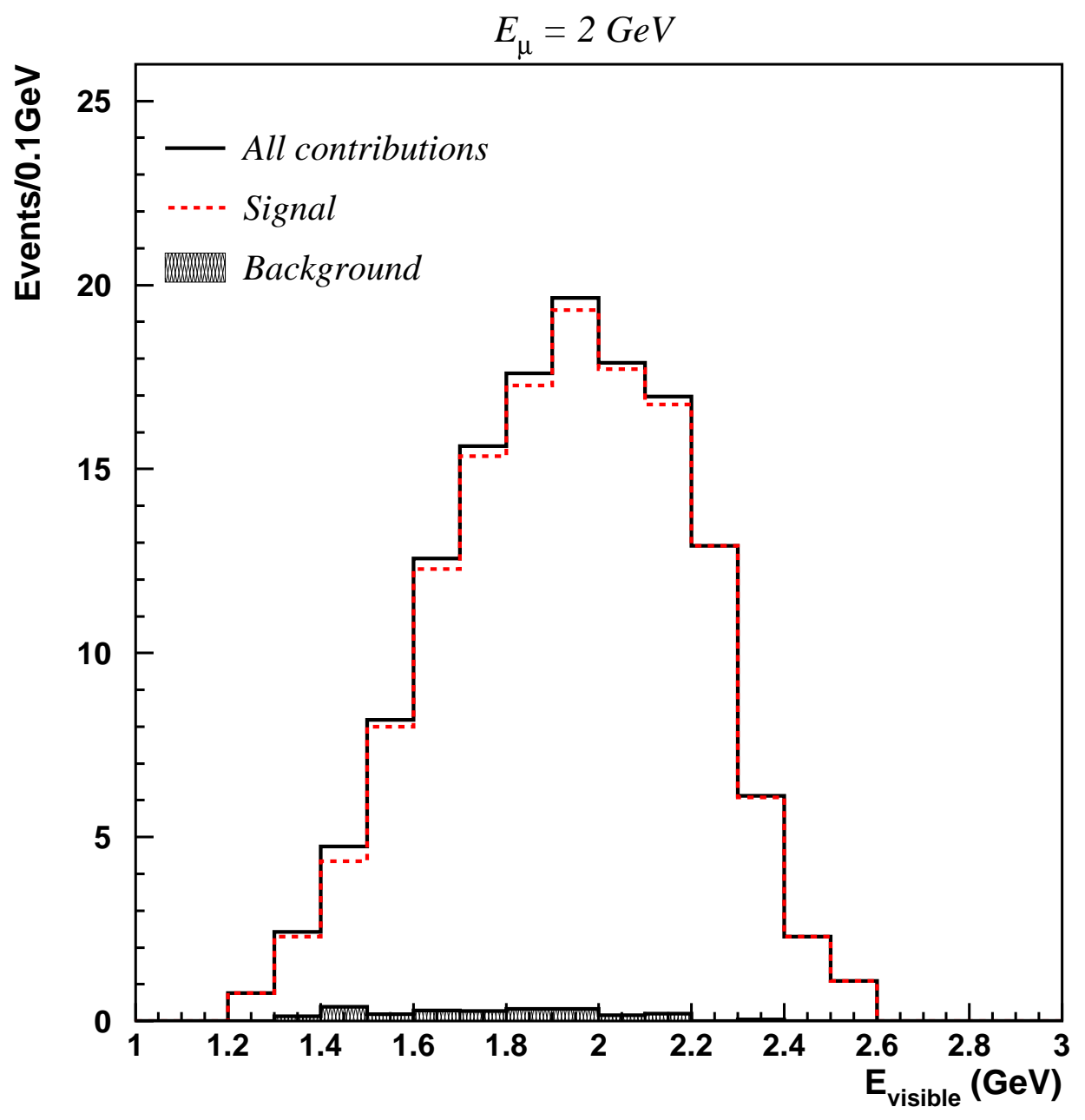

Figure 4: Visible energy distribution (see Figure 3) after a cut on the candidate muon momentum for LFV signal (see text) and backgrounds expected in a detector with the characteristics of an ICARUS $L A r$ TPC. 